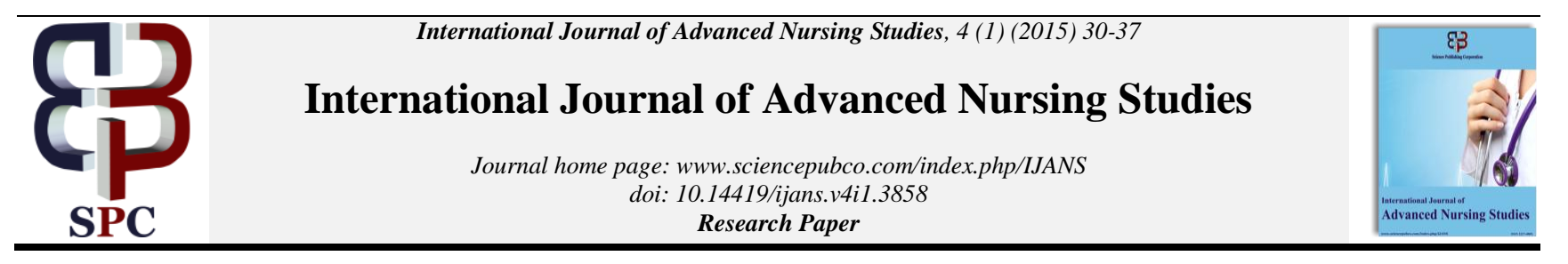

\title{
Nurses' perceptions about child abuse
}

\author{
Ahmad Rajeh Saifan ${ }^{1 *}$, Intima A Alrimawi ${ }^{2}$, Ibraheem Bashayreh ${ }^{3}$ \\ ${ }^{1}$ Assistant Professor, School of Nursing, Applied Science Private University, Amman, Jordan \\ ${ }^{2}$ Assistant Professor, College of Nursing, Birzeit University, Palestine \\ ${ }^{3}$ Assistant Professor, School of Nursing, Philadelphia University, Amman, Jordan \\ *Corresponding author E-mail: a_saifan@asu.edu.jo
}

\begin{abstract}
Background: Despite the efforts to protect children around the world, child abuse and neglect remain serious and global problems. In Palestine, child abuse is hidden under the community culture, does not appear in the Ministry of Health official reports, and little is known about nurses' perceptions towards this phenomenon.

Objectives: To identify nurses' perceptions about child abuse definition, whether they faced such cases during their work, and how they managed them.

Methods: Data were collected using descriptive survey (exploratory) approach. A total of 84 nurses from a major hospital in Ramallah city in Palestine were surveyed.

Results: Only 33\% of the participants intend to use referral system in co-operation with the Ministry of Social Affairs, child protection organizations, or the police in Palestine to deal with child abuse cases. The most seen abuse case was neglect (79\%), followed by psychological abuse (61\%), then physical abuse (57\%) and the least seen was sexual abuse (27\%).

Conclusion: Most of the participants do not know how to deal with child abuse effectively. This research provides baseline information for understanding nurses' practice in the ground, and it helps in presenting the appropriate conditions that enable nurses to fully practice their role toward such cases.
\end{abstract}

Keywords: Child Abuse; Arab; Nursing; Palestine.

\section{Introduction}

Despite the efforts to protect children around the world, child abuse and neglect remain serious and global problems. In the US more than three million children have been exposed to either abuse and/or neglect (Currie and Spatz Widom 2010). The World Health Organization announced that more than 40 million children worldwide are considered victims of child abuse yearly (Piltz and Wachtel 2009). The concept of child abuse varies from one context to another, and there is still a need for a uniform definition (Paxson and Haskins 2009). It was defined by the World Health Organization comprehensively as:

All forms of physical and/or emotional ill-treatment, sexual abuse, neglect or negligent treatment of commercial or other exploitation, resulting in actual or potential harm to the child's health, survival, development or dignity in the context of a relationship of responsibility, trust or power (World Health Organization 2009, p.7).

Child abuse is associated with several factors such as children with disabilities, low socioeconomic status, younger age, and problems within the family (Hendricks et al. 2014, Paxson \& Haskins 2009, Ryan 2003). Stirling and Amaya-Jackson (2008) reported that the youngest children had higher rates of victimization and that girls are more likely to be abused than boys. Chihak (2009) explained that children are mostly abused by someone they know, such as a parent or a day care provider. These factors seem to be consistent among different countries. However, most of the above studies were conducted in the developed countries. It is important to consider that the link between these risk factors and abuse is only statistical association, and not necessarily causal (World Health Organization 2009). It was indicated that child abuse is more common in poor and low social class families. However, child abuse may exist in wealthy and high socioeconomic status families.

Another factor that should be considered when discussing child abuse is the community culture. Child abuse is a complex and context-bound phenomenon that exists in every society, but perceptions about it vary (Lagerber 2001, Pearce \& Pezzot-Pearce 2013). Every culture has a set of behaviors that they consider abusive (Tomison 2007, Alrimawi et al. 2014). The difference between perceptions of child abuse might be obvious when comparing Western culture with Eastern ones. For instance, the power within the Eastern family structure lies solely with the parent; children's rights and opinions are ignored. For example, physical punishments might be seen as extreme by Western culture, but are commonly seen as a necessary prerogative of parents in Eastern countries, representing not only acceptable behavior but even an expression of parental love and caring (Yang 2009). Consequently, it is quite important that health professionals understand the cultures of the children and their families, and they have to develop plans and actions to protect children. These plans should be appropriate to their culture (Gilligan and Akhtar 2006).

Child abuse has serious consequences at both the personal and community levels. This includes the physical and psychological consequences on the abused child (Keane \& Chapman 2008, Reijneveld et al. 2008). Child abuse is also associated with the likeli- 
hood of criminal and abusive action from these victims to society in future, such as drug addiction and violence (Thurston 2006). Several studies argued that the effect of abuse trauma and stress on children's development includes physical or biological, behavioural, emotional, cognitive and social developmental aspects (Adams 2006, Watts-English et al. 2006). Lee and Hoaken (2007) found that there is a significant relationship between child abuse and the development of aggression among children.

Efforts should be concentrated on each person who has responsibility in child protection, such as school teachers, social workers, law enforcement personnel, counsellors and health professionals (Tomison 2007). Health professionals make a major contribution in the recognition and response to child abuse (Gilbert et al. 2009). Of these professionals, nurses have a greater responsibility in this matter because they form the first line of contact with children and their families (Chihak 2009, Piltz \& Wachtel 2009). Nurses have legal and ethical obligations to report any suspected abuse cases (according to their code of conduct), and to provide the appropriate resources and support for the families (Chihak 2009). Research indicates that training increases nurses' awareness about this phenomenon, and improves their ability to recognize the signs and symptoms of child abuse, and consequently increase the likelihood of reporting any suspicious cases (Sidebotham et al. 2007).

In Palestine, as in the other Middle-Eastern countries, nursing is newly developed as a profession. All nurses are educationally prepared with a degree from either university or college, and have to get registration to be able to practice their nursing job (Shukri 2005). They follow international codes of conduct for nursing practice. This includes nurses' responsibility and accountability for their practice and their role in ensuring patient safety, dignity and rights (International Council of Nurses (ICN) 2005). However, nursing practice in the protection of clients and advocating their rights faces challenges. In many occasions, nurses' moral reasoning and ethical decision-making process is inhibited by cultural norms and beliefs (Oweis and Diabat 2005). This might result from lack of training and deficiency of resources, the absence of adequate law enforcement, and lack of emphasis on these codes of conduct during their practice.

Data from surveys about violence against children in Palestine show that the child abuse phenomenon seems to be widespread in Palestinian society (Halileh \& Abdullah 2009, Palestinian Central Bureau of Statistics 2007). However, it is hidden under the community tradition and culture, and does not appear in the Ministry of Health official reports (Halileh and Abdullah 2009). This study aimed to identify nurses' perceptions about child abuse definition, whether they faced such cases during their work, and how they managed them.

\section{Methods}

\subsection{Study design}

This study used non-experimental descriptive cross-sectional design. This design was selected because of the absence of baseline numerical information about this topic in Palestine (Halileh and Abdullah 2009). A structured questionnaire was used to collect the data. This questionnaire was previously used in a Palestinian study (Halileh and Abdullah 2009). Adopting a quantitative approach helped to overcome the sensitivity of the study and made the participants comfortable to discuss this subject. With simple language usage to match the level of education of participants, and careful design, the self-completion questionnaire method can be useful and informative (Polit and Beck 2010).

\subsection{Setting and sample}

The convenience sampling technique was selected in this study. This non-probability technique is flexible and easy to be implemented (LoBiondo-Wood and Haber 2002). The sample included the nurses who have contact with children during their work, and those who were working on a full-time basis.

The current study was conducted in a major tertiary governmental hospital in Ramallah city, Palestine. People from different socioeconomic classes come from all Palestinian cities to seek medical care in this hospital. Around half of the healthcare professionals come from different Palestinian areas to work in this hospital. Therefore, the sample will be representative of the population, which supports the generalizability of the results.

The nurses who worked in the departments where nurses deal with children, including the Paediatric Ward, Intensive Care Unit, Day Care Unit, Medical Ward, Surgical Ward and the Emergency Department, were asked to fill in the self-completed questionnaire. From the 170 nurses who worked in this hospital, 100 nurses who met the inclusion criteria were selected to complete the questionnaire. The response rate was $86 \%$. Two participants were excluded as major parts of the data were missed. Finally, 84 nurses completed the questionnaire and formed the sample of the current study.

\subsection{Ethical consideration}

Ethical approval was obtained from the Ministry of Health in Palestine, and research setting hospital managers. The participants autonomously decided to voluntarily participate in the study. Informed consent was signed by each participant before filling in the questionnaire. The study purposes were explained for each participant. The participants' responses were identified by the questionnaire number in the computer, so their privacy and confidentiality was protected, and nobody outside the research team was informed of their involvement.

\subsection{Instruments and data collection procedure}

The data was collected over a period of six months. Each nurse received a full explanation about the study and its aims. The nurses were asked to complete the questionnaire during their break or when they got back to their homes. Three volunteer nurses helped in recruiting nurses and in the questionnaire completion.

The study questionnaire was derived from a study implemented in Palestine (Halileh and Abdullah 2009). Permission to use this questionnaire was obtained from the study authors. This questionnaire mainly focuses on examining participants' perceptions and attitudes toward child abuse and neglect. It is also checking whether the participants have experience of dealing with suspected child abuse incidents and the way in which they dealt with these incidents (Halileh and Abdullah 2009).

The final version of the study questionnaire comprises three parts: the demographic data, nurses' perceptions of child abuse definition, and whether they experienced suspected cases of child abuse and how they managed it. Perception of child abuse were rated on a scale of (always agree, mostly agree, sometimes agree, and do not agree), with a space for participants' additional comments. The questions regarding whether nurses' had ever seen suspected cases of child abuse were answered with either 'yes' or 'no'. In addition, open questions were used in most of the demographic and personal characteristic questions, such as place of residence, level of education and years of nursing experience. Open questions were also used to provide a space for further exploration and comments after each section. At the end of the questionnaire there were questions about the existence of a protocol and reference system usage that could be answered either with 'yes' or 'no'. Minor changes were performed to the basic adopted questionnaires. For example, for the question about the reference system usage by nurses ("in case there is referral system that would follow-up cases for child abuse and neglect if identified, in cooperation with the ministry of social affairs, child protection organizations, and the police in Palestine, do you use it?"), "in case" was removed as it can insinuate to the participants that such a system does not exist (Halileh \& Abdullah 2009). As previously mentioned, the questionnaire was already used to assess a Palestinian 
population; therefore it was already translated to Arabic language. Nevertheless, it was double-checked by professional translators in Palestine to be sure that the English and Arabic versions are compatible.

\subsection{Data analysis}

All data were analysed by SPSS software version 16 (SPSS Inc, Chicago, Illinois). Most of the data were analysed using descriptive statistics as it is aimed to fill the gap in the numerical data in this area. Nurses' perception of child abuse was analysed using means and standard deviation, while the barriers to child abuse reporting and identification and the open question were analysed using frequencies and percentages (Kirkpatrick and Feeney 2009). Mann-Whitney (non-parametric) test was used to assess nurses perceived barriers to child abuse identification based on certain characteristic among them (including level of education, experience in nursing practice and seniority in the department). This was done as a result of the available sample size and the presence of two categories in some occasions such as junior nurse and senior or head nurse. This may make the normal distribution of the sample questionable. In all of these analyses, a $\mathrm{p}$ value of less than 0.05 was considered statistically significant. Those characteristics were chosen as many literatures reflect the important of them on the nurses' perception of child abuse and their perceived barriers to its identification (Nayda 2004, Lee \& Hoaken 2007, Piltz and Wachtel 2009).

\subsection{Validity and reliability}

Validity refers to the degree to which questionnaires measure what they are structured to measure (Coughlan, Cronin et al. 2007). Reliability means the repeatability of the instrument. Reliability is usually checked by measuring the internal consistency of the instrument. In this research, the authors examined the internal consistency by measuring the Cronbach's alpha. This assesses the correlation between questionnaire items, and it was found to be (.55). Although this number does not reflect high reliability, it reflects acceptable reliability (Peat 2001).

Two factors were considered to optimize the validity of the current study. Firstly, the questionnaire had been used before in a peerreviewed, published research paper, and the content of this questionnaire was covered thoroughly in the literature reviews (Halileh and Abdullah 2009). Secondly, a pilot study had been conducted among a sample of ten nurses to ensure that respondents understand the questions. This was aimed to identify any practical problems in the questionnaire, and to test their reaction to certain questions, which contributes to ensuring the quality of the gained data (Peat 2001)

\section{Results}

\subsection{Participants' characteristics and demographic data}

Eighty-four questionnaires were completed and returned (a response rate of $84 \%$ ). The final sample included 15 nurses from the Intensive Care Unit, 22 from the Emergency Department and 12 from the Medical Ward, and 14 from the Surgical Ward, 13 from the Paediatric Ward and 8 from the Day Care Unit. More than half of the study samples were male $(57 \%)$ and the mean age for the sample was about 28 years (S/D: 6.4, range: 20-49). Around $48 \%$ of the surveyed nurses were permanently live in Ramallah and the others were from elsewhere in the Palestinian Territories (52\%). Around two-thirds of the participants had bachelor's degrees or more, and $38 \%$ of them had a diploma of two years studying. The majority of them $(82 \%)$ held junior nursing positions in their departments.

The results showed that $50 \%$ of the participants were either currently working in or had worked in the paediatric ward before. The mean participant experience in paediatric wards was 1.6 years
(S/D: 3.6, Range: 0-23), while their mean experience in general nursing was 5.7 years (S/D: 5.4, Range: 1-24), which means that $51 \%$ of them had more than four years' of experience in general nursing. The results also showed that none of the nurses had been in a training session about child abuse. Most of the nurses (88\%) stated that there is no policy to deal with child abuse incidences in the hospitals.

Only $33 \%$ of the participants intend to use referral system in cooperation with the Ministry of Social Affairs, child protection organizations, or the police in Palestine to deal with child abuse cases. The nurses raised some reasons for not referring the child abuse cases, including the absence of clear protocol for referring (N: 19), lack of knowledge about the referral system (N: 13), and the thought that the child protection system in Palestine is ineffective (N: 7).

\subsection{Witnessing child abuse cases and their management}

In response to the question about having seen child abuse cases, the most seen abuse case was neglect (79\%), followed by psychological abuse $(61 \%)$, then physical abuse $(57 \%)$ and the least seen was sexual abuse (27\%) (Table 1). Only 64 nurses answered the question about how they managed child abuse cases if seen. However, their response to this question reflected that nurses' management of abuse cases varies according to the type of abuse (see Figures 1 to 4 ).

For physical abuse, the most used line of action was: talking to parent or family member (24\%), informing direct supervisor (if any; $20 \%)$, talking to the child (18\%), and the least used action was to keep quiet/do nothing (11\%) (Figure 1). For sexual abuse, the actions were report to police (28\%), keep quiet/do nothing $(21 \%)$, refer to specialist $(20 \%)$ and the least used action was talking to the child (7\%) (Figure 2).

Looking at the line of actions for psychological abuse showed that the most-used actions were talking to the child (34\%), talking to parent/family members (27\%), referring to a specialist (14\%) and the least used method was to report it to police (2\%) (Figure 3). On the other hand, in child neglect the majority of participants would talk to parents/family members $(41 \%)$, keep quiet/do nothing $(18 \%)$, talk to the child $(16 \%)$, and few participants would refer such cases to specialists (4\%) (Figure 4).

From those 64 participants who answered this question, it is remarkable that eight nurses (12.5\%) stated that they would do nothing for any kind of abuse. Furthermore, in response to the question about their rationale for doing nothing, almost half of them stated that their reasons were: do not want to interfere, fear of consequences and complying family desire. Very few stated that their reasons were: they cannot do anything, and do not know what to do.

Table 1: Nurses Who Have Seen Suspected Cases of Child's Abuse

\begin{tabular}{lll}
$\begin{array}{l}\text { During your practice, have you ever seen a child } \\
\text { with signs/symptoms of suspected abuse or } \\
\text { neglect }\end{array}$ & $\begin{array}{l}\text { Yes/ } \\
\text { Frequencies }\end{array}$ & Percent \\
\hline Physical abuse & 48 & 57.1 \\
Sexual abuse & 23 & 27.4 \\
Psychological abuse & 51 & 60.7 \\
Neglect & 66 & 78.6
\end{tabular}

\subsection{Nurses' perceptions of child abuse}

Participants' response to the question about their perception of the definition of child abuse was coded as follows: do not agree (1), sometimes agree (2) mostly agree (3) and always agree (4), and then the mean, percentage, and standard deviation of their responses were statistically calculated. The higher mean (towards the score of 4) indicated more agreement of the participant that the scenario was abusive. The histogram in Figure 5 indicates that from the 12 child abuse scenarios, the means of nurses' perceptions about child abuse definition were as follows (in descending order): touching the child's body by an adult person (M: 3.83, S/D 0.58 ), not sending child to school in order to help the mother or 
father (M: 3.77, S/D: .65) and talking badly about the child to others and in front of him/her (M: 3.74, S/D: .64). Pushing or shaking the child (M: 3.35, S/D: .83), hitting child with the hand (M: 3.08, S/D: .92), yelling at the child (M: 3.07, S/D: .97) and not controlling the child's exposure to TV and internet had the lowest means (M: 2.71, S/D: 1.15). However, the high standard deviation (S/D 1.15) that can be seen in participants' responses to the "not controlling child exposure to TV and internet" scenario indicates the variation in responses; some of them agreed, others disagreed.

Nurses managmnet to sexual abuse cases

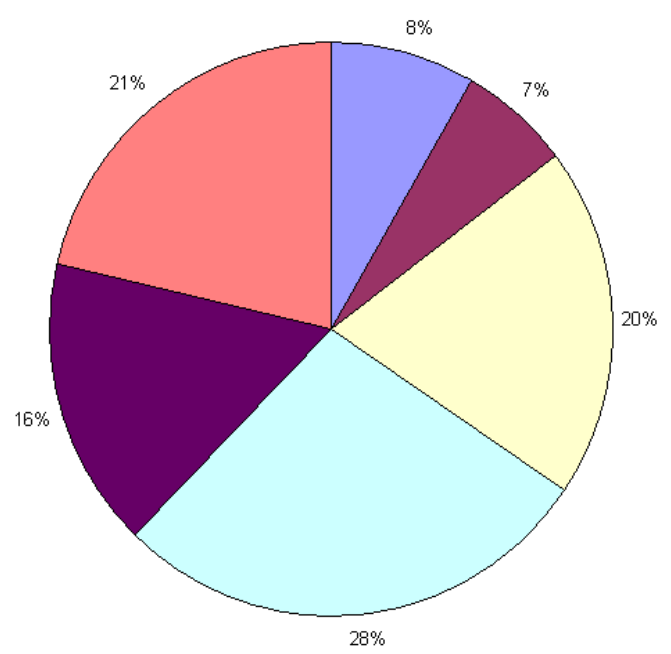

Fig. 1: Nurses Self-Reported Management of Seen Physical Child Abuse Cases

Nurses management to physical child abuse cases

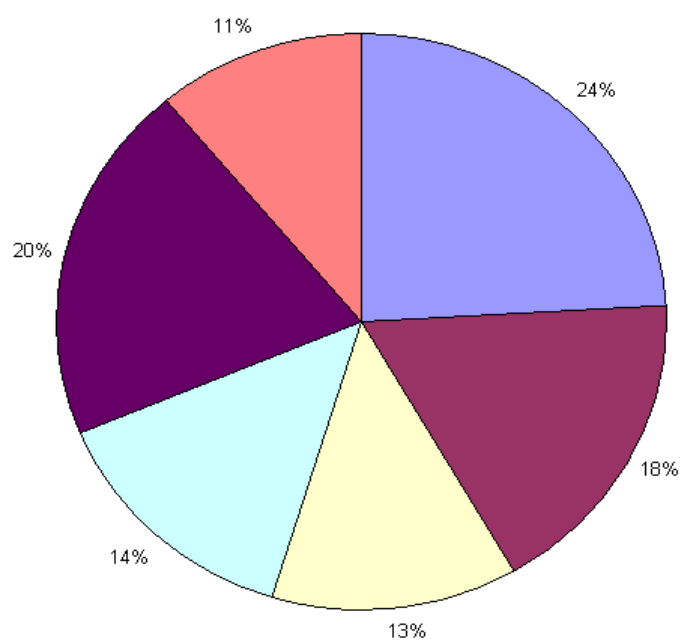

Talking to parents ffamily members

a Talking to child

$\square$ Refer to specialist

$\square$ Report to police

- Inform direct supewisor if any

$\square$ Keep it quiet / do no thing

Fig. 2: Nurses Self-Reported Management of Seen Sexual Child Abuse Cases 
Nurses management to child neglect cases

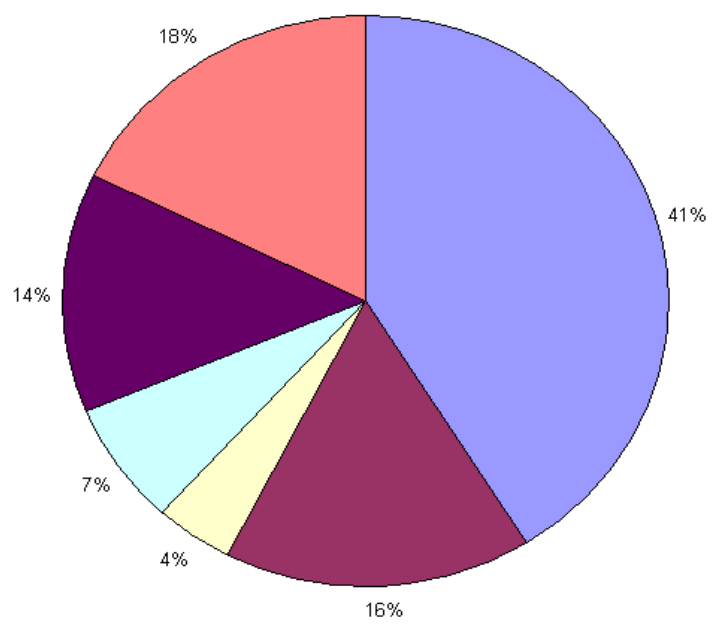

$\square$ Talking to parents /family members
$\square$ Talking to child
$\square$ Refer to specialist
$\square$ Report to police
a Inform direct superisor if any
$\square$ Keep it quiet / do no thing

Fig. 3: Nurses Self-Reported Management of Seen Psychological Child Abuse Cases

Nurses management to psychological abuse cases

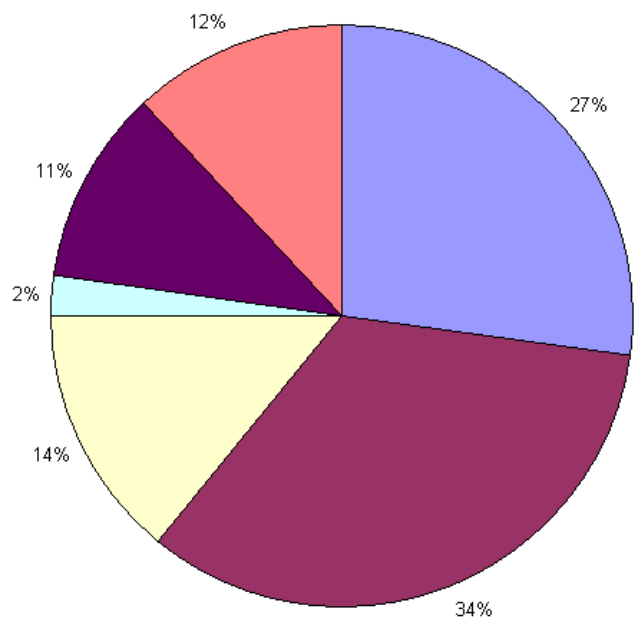

Talking to parents /family members

a Talking to child

$\square$ Refer to specialist

$\square$ Report to police

Inform direct supervisor if any

$\square$ Keep it quiet / do no thing

Fig. 4: Nurses Self-Reported Management of Seen Child Neglect Cases

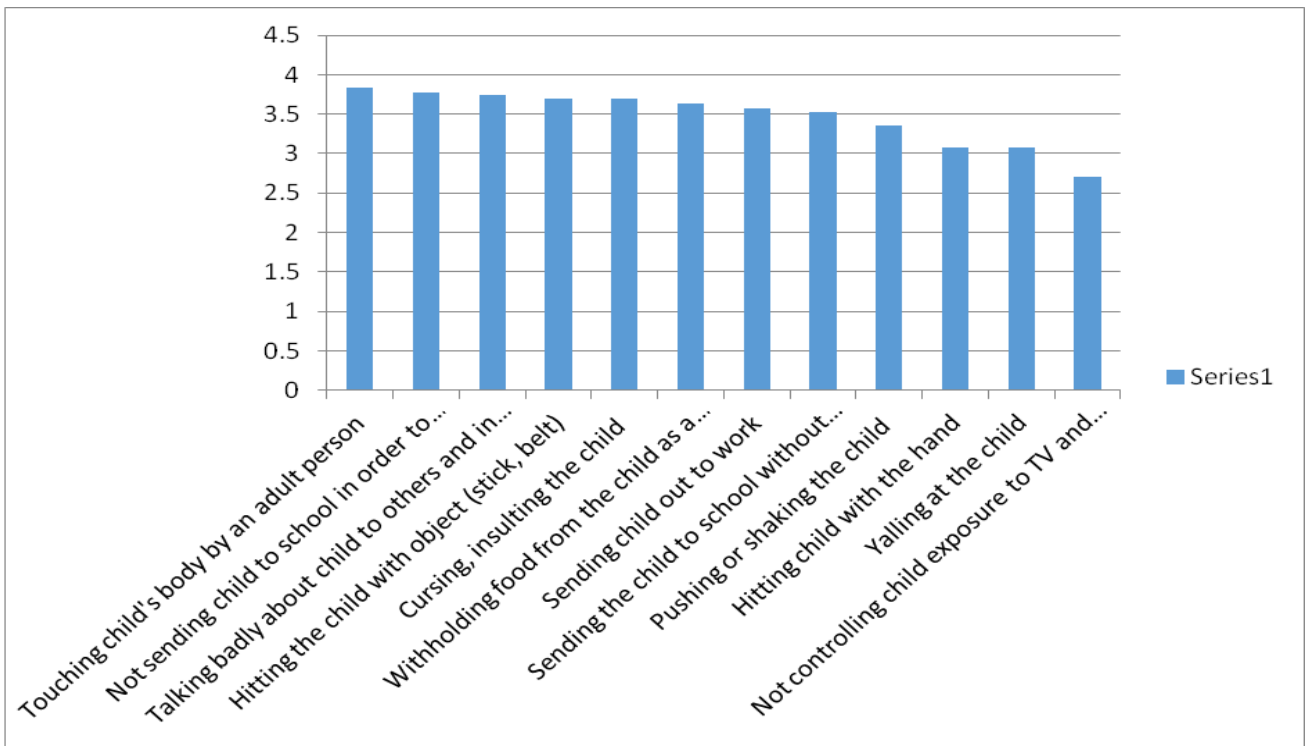

Fig. 5: Mean nurses' perception about child abuse definition

The findings show the effects of certain participants' characteristics (level of education, experience, seniority in the department) on their perceptions of child abuse. Looking at the influence of the level of education on nurses' perceptions indicates that this characteristic significantly affected the perception of the "yelling at the child" scenario (Sig: .01), as $34 \%$ of those with nursing diploma 
education (two years of nursing study) mostly agreed that this is abuse, while $54 \%$ of those with a bachelor's degree or more mostly agreed that this is abuse.

In nursing experience, for the significant effect seen in "sending child out to work" (Sig: .049), 63\% of those with up to four years" nursing experience mostly agreed that this is abuse, while $81 \%$ of those with more than four years' nursing experience mostly agreed that this is abuse. Apparently, seniority in the department affects nurses' perceptions of abuse in many scenarios, including: "sending child out to work" (Sig: .01); 67\% of junior nurses mostly agreed that it is abuse, compared to $100 \%$ of senior nurses or head nurses. For "talking badly about child to others and in front of him/her" (Sing: .049), 78\% of junior nurses mostly agreed that this is abuse, compared to $100 \%$ of senior nurses or head nurses. For "hitting child with the hand" (Sig: .03), 39\% of junior nurses mostly agreed that it is abuse, compared to $73 \%$ of senior nurses or head nurses. Lastly, for "sending the child to school without breakfast" (Sig: .03), 67\% of junior nurses mostly agreed that it is abuse, compared to $93 \%$ of senior nurses or head nurses.

\section{Discussion}

This study is one of the few investigations of Palestinian nurses perceptions of child abuse and neglect. Nurses from different departments of one of the most important hospitals in Palestine were surveyed. More than eighty percent of the surveyed nurses completed and returned the questionnaire. This reflects the nurses' acknowledgment of the importance of this topic. This study was conducted in a tertiary hospital in Ramallah (the capital of Palestine). Healthcare providers come from all over the Palestinian territories to work in this hospital. Patients also come from all the Palestinian areas to seek medical treatment. This may explain the fact that around half of the study sample are from outside Ramallah. This also adds credit to the current study as its sample is representative of the prevailing situation across Palestine. Additionally, conducting this study in one of the developing countries may add to the existing knowledge in this area. The findings of this study highlight some issues on this sensitive subject and provide information to the layout people on the classifications of child abuse and the most seen cases.

This study shows that nurses have insufficient training regarding child abuse and child protection. This was clear as none of the nurses had received any training in this field. Such a lack of nurses' knowledge is consistent with the literature from other countries (Lee et al., 2007; Keane and Chapman, 2008; Chihak, 2009; Starling et al., 2009; Fraser et al., 2009). However, this research argues that Palestinian nurses' lack of knowledge and needs for training can be considered more serious. These findings are also supported by an important study that was conducted in Palestine. Halileh and Abdullah (2009) examined the training needs among physicians by the Community and Public Health Institute. They found that only $30 \%$ of paediatricians, $15 \%$ of general physicians and $10 \%$ of gynaecologists had received training related to child abuse or child protection (Halileh and Abdullah 2009). This means that most of the healthcare providers who directly deal with paediatric clients had no training about child abuse and neglect. This reflects the low priority generally given to this phenomenon, as medical and biomedical aspects of health predominate (Sweileh et al. 2013).

Most of the study participants thought that the main reasons for not referring the child abuse cases are the absence of clear protocol for referring and because they do not know how to refer these cases. This issue was clearly raised in the literature (Sidebotham et al. 2007, Chihak 2009). The absence of policies and protocols to deal with abuse cases was considered to be a major barrier to child abuse identification and reporting (Sidebotham et al. 2007). In contrast, the presence of a clear, concise and structured child protection protocol was thought to facilitate the healthcare providers' mission to deal with such abuse cases (Chihak 2009). This was also thought to improve nurses' awareness, consideration, and documentation of these cases.

In response to the question about seeing child abuse cases, the most seen abuse was neglect (with 79\%), followed by psychological $(61 \%)$ then physical abuse $(57 \%)$, and the least seen was sexual abuse (27\%). This is similar with Halileh's study conducted among Palestinian physicians, which found that $88.7 \%$ of them had encountered cases of abuse during their practices; the mostseen abuse was neglect, and the least-seen was sexual (Halileh and Abdullah 2009). This also concurs with the Palestinian Central Bureau of Statistics survey about violence against Palestinian children, which showed that half of the mothers stated that their children were subject to either physical or emotional abuse (Palestinian Central Bureau of Statistics 2007). On the other hand, MayChahal and Cawson's study, which aimed to provide reliable data about child abuse among adult participants in UK, found that $16 \%$ of participants had experienced maltreatment. Serious forms of it were divided into the following: $7 \%$ physical, $6 \%$ psychological, $5-6 \%$ neglect and $11 \%$ sexual (May-Chahal and Cawson 2005). Comparing the results of May-Chahal and Cawson's (2005) study in the UK with this study suggests that in the former abuse cases are fewer, and that the types of abuse that are most frequently seen differ from those in Palestine. This variance could be connected with many sociocultural factors in the UK, such as the high priority given to child protection generally and at an institutional level (Wilson and James 2002), the deep culture of protection in the UK context (Powell 2007), and the continuous nursing training regarding child protection and abuse phenomena (Baverstock et al. 2008) This study reflects that the management of abuse cases varies according to the type of abuse. This is in agreement with the findings of some earlier studies. For instance, Gilbert et al. (2009) studied the nursing decision-making process in case of dealing with child abuse incidences. Surprisingly, few nurses (12.5\%) from those who responded to the question about child abuse cases' management stated that they will do nothing for any kind of abuse. Half of responses about the reason for that are related to cultural issues (do not want to interfere, fear of consequences, complying with family desire). Recognising cultural needs and practices would result in improving the cooperation and the communication between the healthcare professionals and the patients and their relatives (Blue 2000). All the surveyed nurses were Palestinians. They all understand the influence of culture on people's behaviour. The nurses may avoid intrusion and interference in strong family structures. Some of them may feel that child abuse is a family issue with which they should not interfere. Young and Shami (1997) indicated that kinship is one of the fundamental elements of social organisation in both rural and urban areas, and health behaviour appears to be strongly affected by family structure and household composition. In Palestine, family structure lies solely with the parent; children's rights and opinions are ignored. For example, physical punishments which might be seen as extreme by Western culture can be regarded as acceptable punishments and as an expression of parental love and caring in Eastern culture (Yang 2009). Consequently, it is quite important that health professionals understand the cultures of the children and their families, and develop plans and action that empower and protect children that are appropriate to their culture (Gilligan and Akhtar 2006).

Significantly, the study findings reveal that nurses with more education and experience were more positive in their perception and management of child abuse incidences. For example, $34 \%$ of nurses with diploma education mostly agreeing that "yelling at the child" is abuse, compared to $54 \%$ of those with bachelor's degrees or higher (Sig: .01). Moreover, 63\% of those with up to four years' nursing experience mostly agreed that "sending child out to work" is abuse, compared to $81 \%$ of those with more than four years' nursing experience (Sig: .049). Nursing knowledge and practice have changed rapidly in the last decades and increasing nurse competency is required (Kowitlawakul 2013). All nurses have the responsibility to develop themselves theoretically and clinically to be able to provide the optimal health care services to 
patients and community. This may explain the findings of the current study, as many of the nurses stated that they would do nothing if they suspected a case of child abuse. Most of the surveyed nurses were young (the mean age was 28 years). The vast majority of them have minimal years of experience and they have had no training or education about managing child abuse incidences. Therefore, it is recommended to develop training and education programs to guide the nurses to deal with these incidences.

\section{Conclusion}

This study investigated nurses' perceptions about child abuse definition, whether they face such cases during their work and how they managed these situations. A descriptive (survey) exploratory approach was used to answer the research questions. It was clearly raised that the nurses have some ideas about the child abuse phenomenon and they faced these cases frequently during their practice. The nurses tried to manage these situations They admitted the difficulty of doing this, especially in some incidents such as sexual abuse. Despite the trials of many nurses to deal with these incidents, they do not know how to handle it effectively. This happened as a result of several factors such as lack of training, the absence of clear protocols for dealing with such cases and cultural issues. It was explained that child abuse might be viewed as a private issue and as a part of child rearing process. The current study is pioneering in this area, and it provides baseline information for understanding nurses' practice in child abuse detection and management. It might help in ensuring the availability of the appropriate conditions that enable nurses to fully practice their role in the identification and managing these cases. However, there is a need for further studies about this topic that include the private and non-governmental sector, and involve nurses who work in the primary and tertiary health services.

\section{Acknowledgements}

The authors are grateful to the Applied Science Private University, Amman, Jordan, for the financial support granted to cover the publication fee of this research article. We would like to thank all of those who helped us during our trip for the completion of this research. This study could not have been accomplished without the contribution of the nurses who agreed to take part in this study, who must necessarily remain anonymous.

\section{References}

[1] Adams C. M (2006) The Consequences of Witnessing Family Violence on Children and Implications for Family Counselors. The Family Journal, 334-341 http://dx.doi.org/310.1177/1066480706290342.

[2] Alrimawi I, Saifan A, AbuRuz M (2014). Barriers to child abuse identification and reporting. Journal of Applied Sciences, 14, 2793 http://dx.doi.org/10.3923/jas.2014.2793.2803.

[3] Baverstock A, Bartle D, Boyd B \& Finlay F (2008). Review of child protection training uptake and knowledge of child protection guidelines. Child Abuse Review, 17, 64-72. http://dx.doi.org/10.1002/car.1011.

[4] Chihak A (2009). The nurse's role in suspected child abuse. Paediatrics and Child Health, 19, S211-S213 http://dx.doi.org/210.1016/j.paed.2009.1008.1005.

[5] Coughlan M, Cronin P \& Ryan F (2007). Step-by-step guide to critiquing research. Part 1: quantitative research. British journal of nursing, 16 , 658-663. http://dx.doi.org/610.12968/bion.12007.12916.12911.23681.

[6] Currie J \& Spatz Widom C (2010). Long-Term Consequences of Child Abuse and Neglect on Adult Economic Well-Being. Child Maltreatment, 15 ,

$111-120$ http://dx.doi.org/110.1177/1077559509355316.

[7] Fraser J, Mathews B, Walsh K, Chen L \& Dunne M (2010). Factors influencing child abuse and neglect recognition and reporting by nurs- es: A multivariate analysis. International Journal of Nursing Studies, 47, 146-153. http://dx.doi.org/110.1016/j.ijnurstu.2009.1005.1015.

[8] Gilbert R, Kemp A, Thoburn J, Sidebotham P, Radford L, Glaser D, et al. (2009). Recognising and responding to child maltreatment. The Lancet, $373, \quad 167-180 . \quad$ http://dx.doi.org/10.1016/S01406736(08)61707-9.

[9] Gilligan P \& Akhtar S (2006). Cultural Barriers to the Disclosure of Child Sexual Abuse in Asian Communities: Listening to What Women Say. British Journal of Social Work, 36, 1361-1377. http://dx.doi.org/1310.1093/bjsw/bch1309.

[10]Halileh S \& Abdullah A (2009). Child abuse and neglect services in the occupied Palestinian territory. Eastern Mediterranean Health Journal, 15, 1504-1512.

[11]Hendricks C, Lansford J, Deater-Deckard K \& Bornstein M (2014). Associations between Child Disabilities and Caregiver Discipline and Violence in Low- and Middle-Income Countries. Child Development, 85, 513-531. http://dx.doi.org/510.1111/cdev.12132.

[12] International Council of Nurses (ICN). (2005). The ICN code of ethics for nurses: Switzerland: ICN.

[13] Keane C \& Chapman R (2008). Evaluating nurses' knowledge and skills in the detection of child abuse in the Emergency Department. International Emergency Nursing, 16, 5-13. http://dx.doi.org/10.1016/j.ienj.2007.1011.1006.

[14] Kirkpatrick L \& Feeney B (2009). A simple guide to SPSS: for version 16. Wadsworth: Belmont.

[15] Kowitlawakul Y (2013). From novice to expert: Sharing professional development experience in different practice settings. Singapore Nursing Journal, 40, 43-46.

[16]Lee V \& Hoaken P (2007). Cognition, emotion, and neurobiological development: mediating the relation between maltreatment and aggression. Child Maltreatment, 12, 281-298. http://dx.doi.org/10.1177/1077559507303778.

[17]LoBiondo-Wood G \& Haber J (2002). Nursing research: methods, critical appraisal, and Utilization. Mosby, Philadelphia Palestinian Central Bureau of Statistics. (2007). Children and Domistic Violence. Available at: http://www.pcbs.gov.ps (accessed 10 October 2013).

[18] May-Chahal C \& Cawson P (2005). Measuring child maltreatment in the United Kingdom: A study of the prevalence of child abuse and neglect. Child Abuse \& Neglect, 29, 969-984. http://dx.doi.org/10.1016/j.chiabu.2004.05.009.

[19]Nayda R (2004). Registered nurses' communication about abused children: rules, responsibilities and resistance. Child Abuse Review, 13, 188-199. http://dx.doi.org/10.1002/car.844.

[20]Oweis A \& Diabat K (2005). Jordanian nurses perception of physicians' verbal abuse: findings from a questionnaire survey. International Journal of Nursing Studies, 42, 881-888. http://dx.doi.org/10.1016/j.ijnurstu.2004.11.005.

[21]Paxson C \& Haskins R (2009). Introducing the issue. The Future of Children, 19, 3-17. http://dx.doi.org/10.1353/foc.1350.0034.

[22]Pearce J \& Pezzot-Pearce T (2013). Psychotherapy of abused and neglected children. Guilford Publications, New York.

[23]Peat J (2001). Health science research: a handbook of quantitative methods. Sage, London

[24] Piltz A \& Wachtel T (2009). Barriers that inhibit nurses reporting suspected cases of child abuse and neglect. Australian Journal of Advanced Nursing, 26, 93

[25]Polit D \& Beck C (2010). Essentials of Nursing Research: Appraising Evidence for Nursing Practice (7th Ed.). Lippincott Williams \& Wilkins, China.

[26]Powell C (2007). Safeguarding children and young people: a guide for nurses and midwives. Open University Press, England.

[27]Reijneveld S, de Meer G, Wiefferink C, \& Crone M (2008). Detection of child abuse by Dutch preventive child-healthcare doctors and nurses: Has it changed? Child Abuse \& Neglect, 32, 831-837. http://dx.doi.org/10.1016/j.chiabu.2007.09.014.

[28]Ryan B (2003). Do you suspect child abuse? RN, 66, 73-77.

[29]Shukri R (2005). Status of nursing in the Arab world. Ethnicity \& disease, $15, \mathrm{~S} 1-88-89$

[30] Sidebotham P, Biu T \& Goldsworthy L (2007). Child protection procedures in emergency departments. Emergency Medicine Journal, 24, 831-835. http://dx.doi.org/10.1136/emj.2007.051011.

[31]Starling S, Heisler K, Paulson J \& Youmans E (2009). Child Abuse Training and Knowledge: A National Survey of Emergency Medicine, Family Medicine, and Pediatric Residents and Program Directors. Pediatrics, 123, e595-e602. http://dx.doi.org/10.1542/peds.2008-2938.

[32] Sweileh W, Zyoud S, Sawalha A, Abu-Taha A, Hussein A \& Al-Jabi S (2013). Medical and biomedical research productivity from Palestine, 2002 - 2011. BMC Research Notes, 6, 41. http://dx.doi.org/10.1186/1756-0500-1186-1141. 
[33]Thurston C (2006). Child abuse: recognition of causes and types of abuse. Practice Nurse, 31, 51-56.

[34]Tomison A (2007). Overcoming structural barriers to the prevention of child abuse and neglect: Melbourne: Australian Institute of Family Studies.

[35] Watts-English T, Fortson B, Gibler N, Hooper S \& De Bellis M (2006). The psychobiology of maltreatment in childhood. Journal of Social Issues, 62, 717-736. http://dx.doi.org/10.1111/j.15404560.2006.00484.x.

[36] Wilson K \& James A (2002). The child protection handbook. Edinburgh: Baillière Tindall.

[37]World Health Organization (2009). Child maltreatment. Available at: http://www.euro.who.int/violenceinjury/violence/20051125_1 (accessed 20 December 2013).

[38]Yang S (2009). Cane of love: parental attitudes towards corporal punishment in Korea. British Journal of Social Work, 39, 1540-1555. http://dx.doi.org/10.1093/bjsw/bcn034.

[39] Young, W \& Shami S (1997). Anthropological approaches to the Arab family. Journal of Comparative family studies, 28, 1-13. 